\title{
Transfer Learning from Simulation to Experimental Data: NMR Chemical Shifts Predictions
}

\author{
Herim Han ${ }^{1), 2)}$, Sunghwan Choi ${ }^{1 \text { ) }}$
}

1) Division of National Supercomputing, Korea Institute of Science and Technology Information, 245 Daehak-Ro, Yuseong-Gu, Daejeon, 34141, Republic of Korea

2) Department of Polymer Science and Engineering, Dankook University, 152 Jukjeon-Ro, Suji-Gu, Yongin, Gyeonggi, 16890, Republic of Korea

E-mail: sunghwanchoi@kisti.re.kr 


\section{S1. Detailed description of preprocessing of simulation database}

Step1. Generation of 3D structure from SMILS structures.

We load experimental database by python pickle and extract atomic coordinates from the contained RDKIT Mol objects using EmbedMolecule and GetConformers functions. When calling EmbedMolecule function, we set maximum number of attempts to try embedding as 30 . For the molecule belonging to the test subset, we use arbitrary chosen number for random seed to generate multiple geometries from the same molecule. For some mol objects, EmbedMolecule fails.

Step2. Validation of structure using INCHI

To confirm validity of the converted structures, we compare InChI codes directly from Mol object and from the obtained geometry through Step1.

1. The mol objects from experimental database are directly converted InChI code using Chem.inchi.MolToInchi().

2. The InChI codes from 3D structures are generated through Open babel command, (obabel -i xyz *.xyz -oinchi).

3. For both InChI codes, the part that include connectivity information of molecule is parsed by using sed command (sed -e 's $/ /[a b d-z] . * \$ \mid g)^{*}{ }^{1}$

4. Identity of two parse InChI codes are confirmed. If they are not identical, the generation of 3D geometry is not successfully proceeded.

The cases that are failed on each steps are summarized in Table S1. In our experiments, we use $21,116 / 5,304{ }^{13} \mathrm{C}$ and $9,657 / 2,411{ }^{1} \mathrm{H}$ molecular structures as train set and test sets.

Table S1. Number of cases to fail to pass preprocessing steps

\begin{tabular}{lcc}
\hline & $\begin{array}{c}\text { Number of fails in }{ }^{1} \mathrm{H} \\
\text { database }\end{array}$ & $\begin{array}{c}\text { Number of fails in }{ }^{13} \mathrm{C} \\
\text { database }\end{array}$ \\
\hline Step 1 & 66 & 52 \\
Step 2 & 663 & 435 \\
\hline
\end{tabular}




\section{S2. Statistics of Experimental and Simulation Databases}
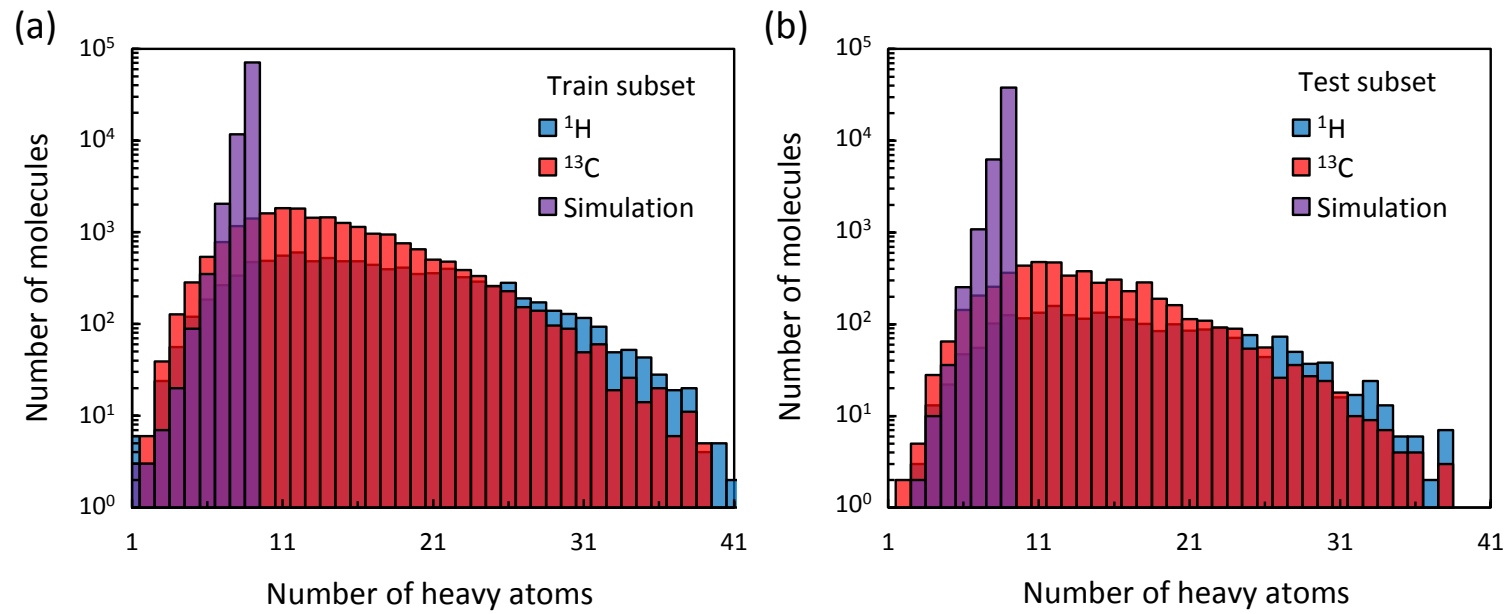

Figure S1. The number of molecules in experimental and simulation databases according to the number of heavy atoms in molecules of (a) train subset and (b) test subset.

(a)

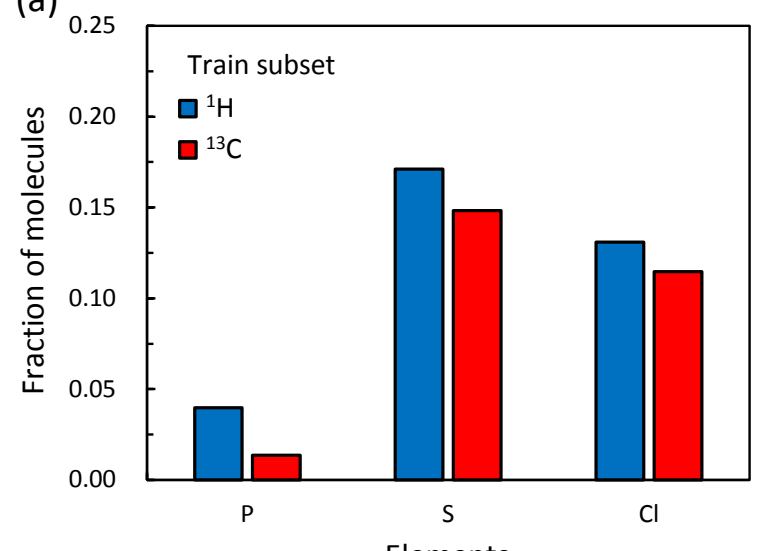

(c)

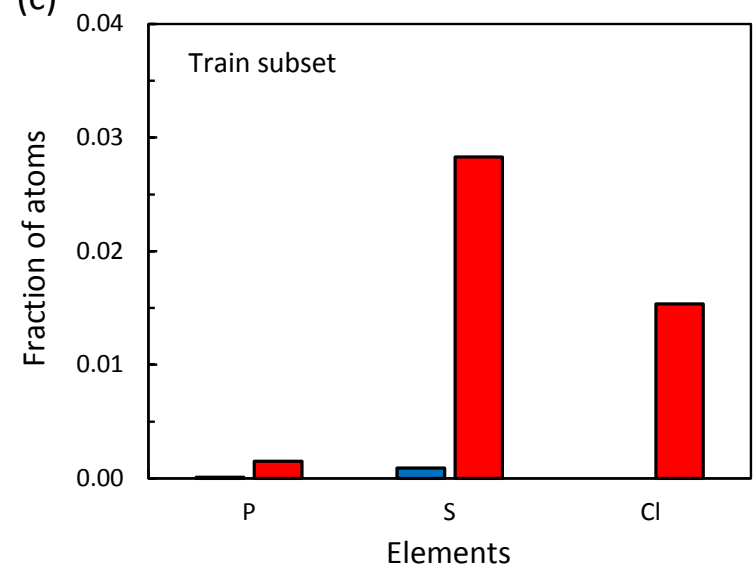

(b)

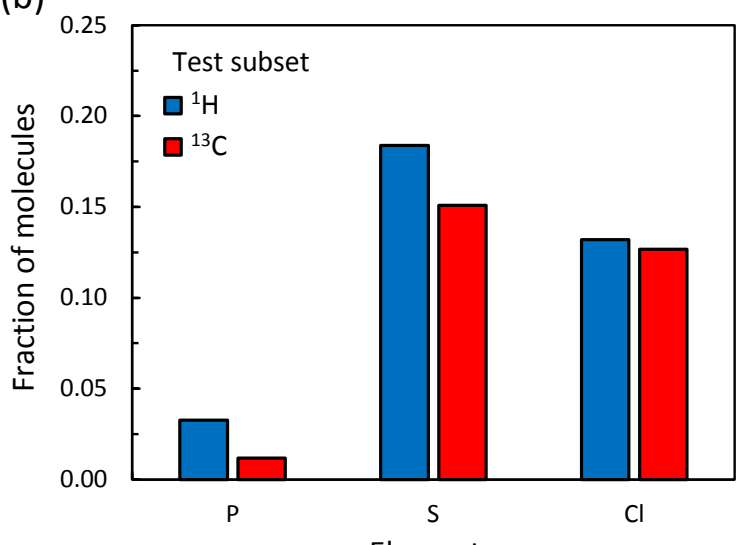

(d)

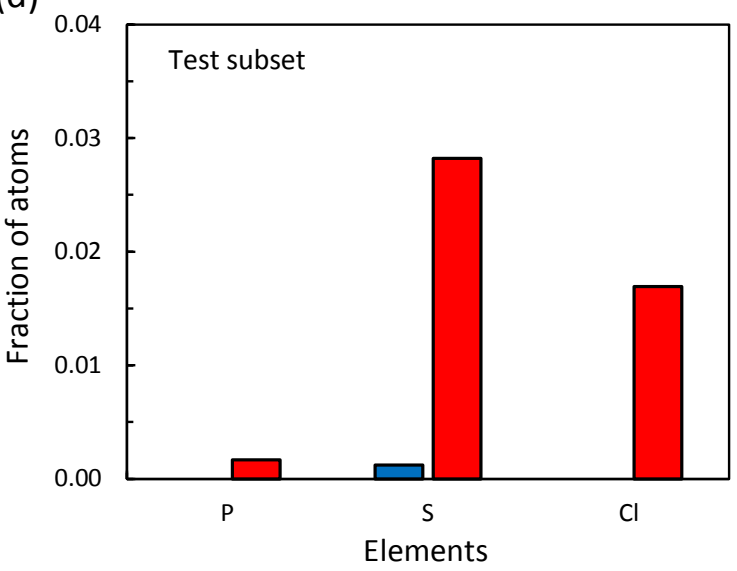

Figure S2. Ratio of molecules containing P, S, and $\mathrm{Cl}$ with respect to whole molecules in (a) train subset and (b) test subset of experimental database. Ratio of NMR-active atoms bonded to P, S, and Cl in (c) train subset and (d) test subset of experimental database. 

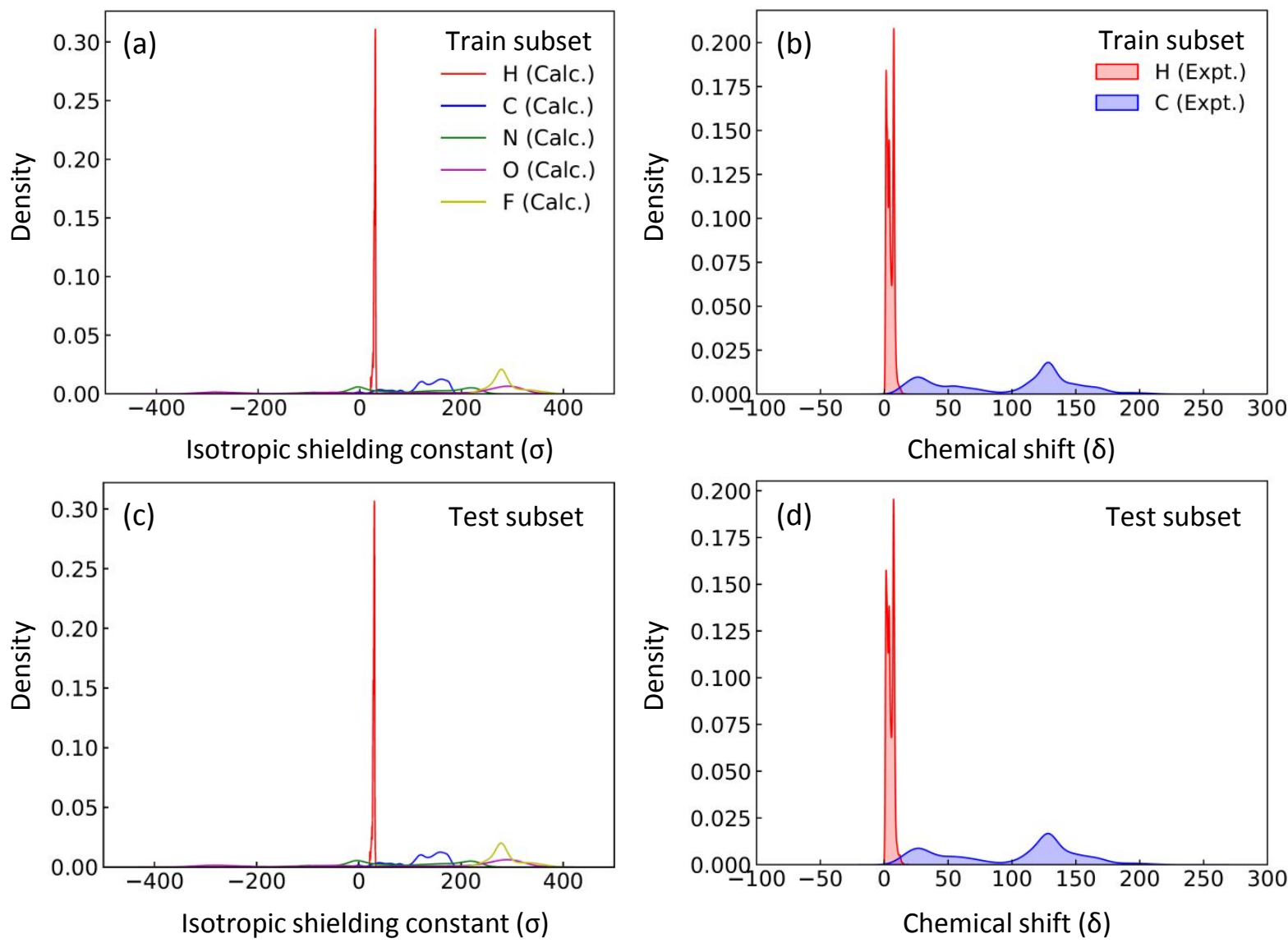

Figure S3. The distribution of isotropic shielding constants and chemical shift values. The isotropic shielding constant for $\mathrm{H}, \mathrm{C}, \mathrm{N}, \mathrm{O}, \mathrm{F}$ in (a) train and (c) test subset. The chemical shift for $\mathrm{H}, \mathrm{C}$ in (b) train and (d) test subset. 


\section{S3. Performance of machine learning model with random sampling}
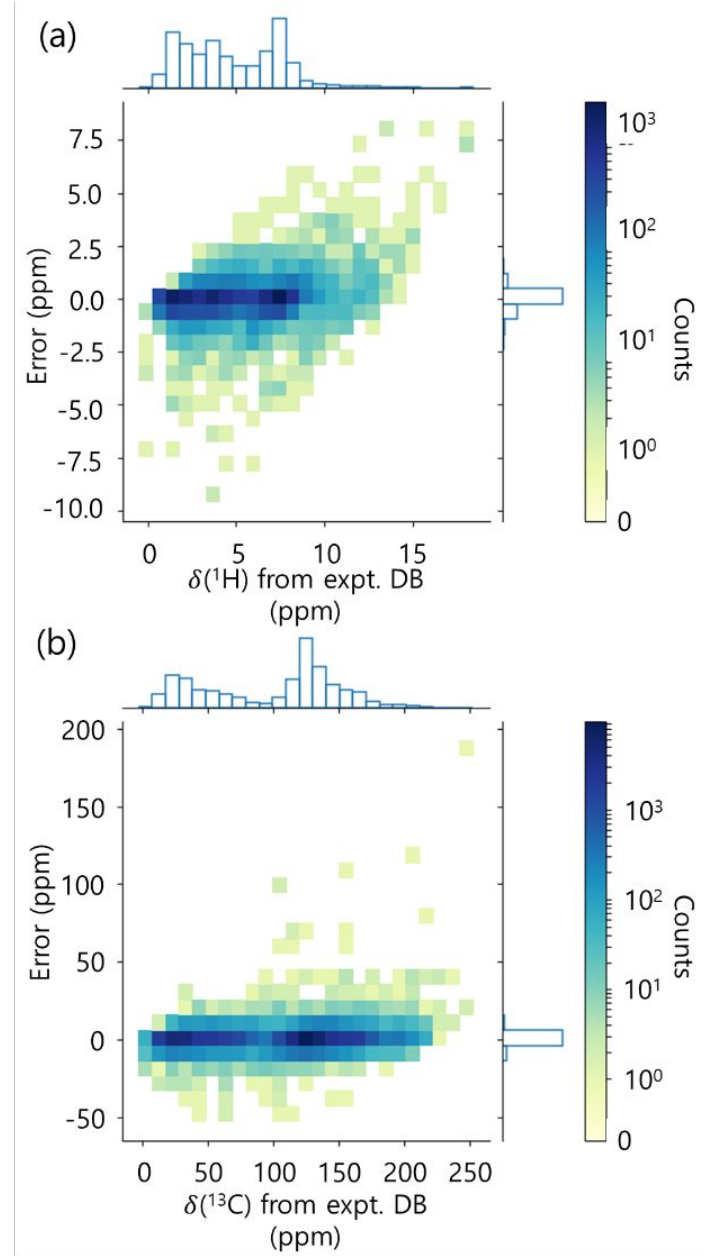

Figure S4. Distribution of prediction error of both (a) $\delta\left({ }^{1} \mathrm{H}\right)$ and $(\mathrm{b}) \delta\left({ }^{13} \mathrm{C}\right)$ that is evaluated from finetuned model with $10 \%$ of random sampling

Table S2. Mean absolute error (in ppm) for test subset of the experimental database from reference model ${ }^{2}$ and ours according to the fraction of used train subset.

\begin{tabular}{ccccc}
\hline $\begin{array}{c}\text { Fraction of } \\
\text { used train } \\
\text { subset }\end{array}$ & \multicolumn{2}{c}{ Expt. (H) } & \multicolumn{2}{c}{ Expt. (C) } \\
\hline $1 \%$ & 0.43 & 1.35 & 4.95 & 6.04 \\
$4 \%$ & 0.37 & 0.41 & 2.64 & 2.97 \\
$7 \%$ & 0.34 & 0.40 & 2.58 & 2.17 \\
$10 \%$ & 0.33 & 0.39 & 2.22 & 2.01 \\
\hline
\end{tabular}




\section{S4. Details of subgroups and target learning results}

(a)



(c)

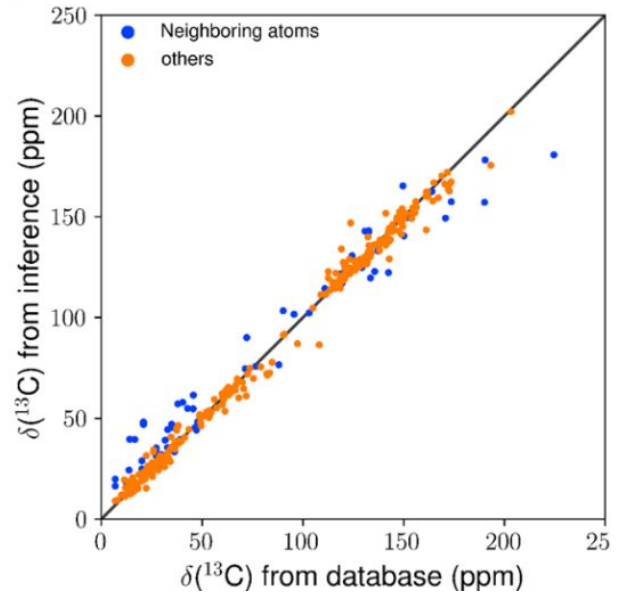

(b)

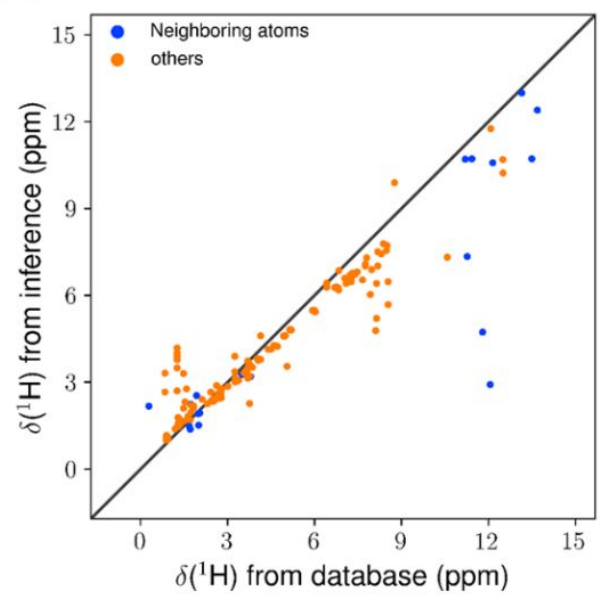

(d)

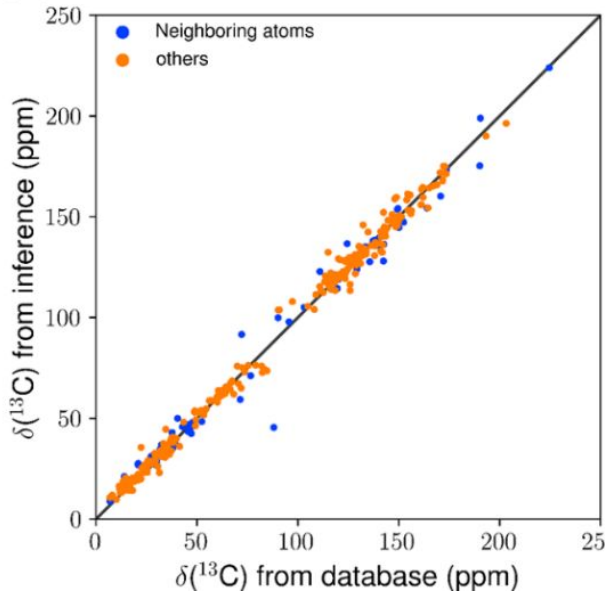

Figure S5. The distribution of true and predicted chemical shift values for $(a) /(b)$ thiol and (c)/(d) phosphate subgroups. The inference values in (a) and (c) are obtained from the model trained with randomly sampled training molecules. The inference values in (b) and (d) are obtained from the model trained with training molecules belonging to the corresponding subgroups.

Table S3. Summary statistics of both subgroups.

\begin{tabular}{clcc}
\hline Subgroup & \multicolumn{1}{c}{ Statistics } & Thiol & Phosphate \\
\hline \multirow{4}{*}{ Training } & No. of molecules & 75 & 358 \\
& No. of NMR-active atoms & 209 & 1403 \\
& No. of neighboring atoms & 373 & 2887 \\
\hline \multirow{3}{*}{ Test } & No. of molecules & 26 & 80 \\
& No. of NMR-active atoms & 70 & 301 \\
& No. of neighboring atoms & 162 & 521 \\
\hline
\end{tabular}




\section{References}

(1) Nakata, M., Shimazaki, T., Hashimoto, M. \& Maeda, T. PubChemQC PM6: Data Sets of 221 Million Molecules with Optimized Molecular Geometries and Electronic Properties. J. Chem. Inf. Model. 60, 5891-5899 (2020).

(2) Jonas, E. \& Kuhn, S. Rapid prediction of NMR spectral properties with quantified uncertainty. J. Cheminform. 11, 50 (2019). 